\title{
Journal of Cancer Biology and Therapeutics Targeting Myc and Hdac8 with a Combination of SiRNAs Inhibits Tumor Growth in a Murine Neuroblastoma Xenograft Model
}

\author{
Prashad $\mathbf{N}^{*}$ \\ Genetics in Medicine, Houston, TX 77054, USA \\ *Correspondence: Nagindra Prashad, Genetics in Medicine, 8285 El Rio St, Suite 150 Houston, Texas 77054, USA, \\ E-mail: Nagindra.prashad@gmail.com
}

Received date: November 11, 2019; Accepted date: January 20, 2020; Published date: January 25, 2020

\begin{abstract}
Neuroblastoma is a common tumor of the peripheral nervous system in children. Highly aggressive MYC-driven neuroblastoma is defined by increased MYC and/or MYCN expression. HDAC8 overexpression is associated with advanced neuroblastoma. Previously, we have demonstrated that transient knockdown of both Myc and Hdac8 using siRNA significantly suppressed neuroblastoma cells proliferation compared to knockdown of either target in vitro. In this study, we further investigated whether combinational targeting Myc and Hdac8 in neuroblastoma xenograft mice model is consistent with our previous findings. Intratumoral treatment with siRNA-MYC and siRNA-HDAC8 reduced the levels of the target MYC protein by $64 \%$ and HDAC 8 by $85 \%$; in addition, we found that the average tumor growth was reduced by $80 \%$ compared to that of control tumors treated with NC-siRNA. Our results suggest the potential therapeutic effect of the combination of siRNA-MYC and siRNA-HDAC8 for neuroblastoma treatment.
\end{abstract}

Keywords: Neuroblastoma, Myc, Hdac8, siRNA, xenograft tumor, siRNA-Hdac8, siRNA-Myc, A/J mice

Abbreviations: MYC: Myelocytomatosis; HDAC8: Histonedeacetylase8; siRNA: small inhibitory RNA; siRNA-Myc: siRNA specific for Myc mRNA; siRNA-Hdac8: siRNA specific for Hdac8 mRNA; A/J mice: Syngeneic Jackson lab mice

\section{Introduction}

Neuroblastoma is the most frequently diagnosed extracranial solid tumor in children. About $90 \%$ of cases occur in children less than 5 years old and it is rare in adults. Of cancer deaths in children, about $15 \%$ are due to neuroblastoma [1]. Chances of long-term survival, however, are less than $40 \%$ despite aggressive treatment [2].

MYC is an oncogenic transcription factor that is overexpressed in many types of cancer. MYC has been shown to directly upregulate a protumorigenic group of miRNAs and represses several suppressor miRNAs, thus contributing to tumorigenesis [3]. For example, MYC overexpression can upregulate the oncogenic miR-17-92 cluster, that are directly activated in lymphoma [4], and can also repress several suppressor miRNAs [3]. The MYC gene is amplified in various human cancers, including in lung carcinoma, breast carcinoma, and colon carcinoma [5].

Histone deacetylases affect gene expression by altering the histone acetylation status, and that as a consequence, HDAC overexpression contribute to tumorigenesis by affecting the expression of key mRNAs and miRNAs. HDACs are overexpressed in most cancers, leading to histone deacetylation, inhibition of growth- 
suppressive genes, and increased cell proliferation [6]. HDAC8 overexpression correlates with advanced neuroblastoma in patient tumor samples, and HDAC8 inhibition reduced cell proliferation and induced neuroblastoma cell differentiation [7]. HDAC inhibitors reduced the proliferation and induced the apoptosis of neuroblastoma cells in vitro and in vivo in mice $[8,9]$.

Given that both MYC and HDACs play an important role in the maintenance of the normal cellular physiological functions and that their overexpression is linked to neuroblastoma tumorigenesis, we asked whether the levels of both MYC and HDAC8 should be reduced to obtain significant inhibition of cell proliferation.

In a previous study, we investigated the effects of siRNAs targeting Hdac8 and Myc in murine neuroblastoma cells. RNA interference is a process of posttranscriptional gene silencing in which a double stranded RNA inhibits gene expression in a sequence-dependent manner via degradation of the corresponding mRNA. Small interfering RNAs (siRNAs) can be used as potent and specific tools for gene knockdown. Several laboratories have reported siRNA targeting of gene expression in cancer cells and the inhibition of cell proliferation in vitro and tumor growth in vivo [10-13].

We previously found that in vitro, single-agent siRNAHDAC8 or SiRNA-MYC inhibited cell proliferation by 40 $50 \%$; however, treatment with the combination of siRNAMYC + siRNA-HDAC8 inhibited cell proliferation by $86 \%$ [14]. To further confirm these findings in an animal model, we set out to verify if tumor growth can be inhibited in a neuroblastoma xenograft mouse model when tumors are treated with a combination of siRNA-MYC and SiRNAHDAC8.

\section{Materials and Methods}

\section{Reagents}

Cell culture media, DMEM with high glucose (D6429), essential and non-essential amino acids (M5550, m7145), Bt2c AMP (D0627), the colorimetric Caspase 3 kit (Code CASP-3-C), were purchased from Sigma Aldrich, St. Louis. Fetal bovine serum (FBS) was purchased from Phenix Research Products, Candler, NC, USA. BD-Falcon tissue culture 96-well plates (353072) were purchased from BD Biosciences.

The MTS Cell Titer 96 Aqueous One Solution (Cat \# G3580) cell proliferation assay was purchased from
Promega Biotechnology, Madison, WI, USA.

Corning 96-well EIA/RIA plates (CLS3369) were used for ELISA. Antibodies for HDAC 8, H-145 (sc11405), CMYC, C-19 (SC-786), were purchased from Santa Cruz Biotechnology, Dallas, TX, USA.

Negative control \#2 siRNA (\#4390846), siRNA-HDAC 8 (S88696), siRNA-MYC (S70224) and Lipofectamine RNAi Max (\#13778075) were purchased from Life Technologies/ Ambion/ Invitrogen.

A/J female mice 6 weeks old were purchased from Jackson lab, Bar Harbor, Maine, USA

Mice were housed in animal facility at Nanospectra Biosciences Inc. Houston, Texas

\section{Cells and cell culture}

Mouse neuroblastoma cholinergic clonal cells (S20) were obtained from Dr. Marshall Nirenberg of The US National Institutes of Health (NIH). Cells were grown in monolayers in DMEM supplemented with essential and nonessential amino acids, penicillin/streptomycin, and $10 \% \mathrm{FBS}$ at $37^{\circ} \mathrm{C}$ with $5 \% \mathrm{CO}_{2}$ and humidity.

\section{Neuroblastoma tumor model}

Mice experiments were performed with the approval of the institutional Animal Care and Use Committee, IACUC at Nanospectra Biosciences Inc. Houston, Texas.

A/J female mice six weeks old were purchased from Jackson Laboratory, Bar Harbor, Maine, USA.

Murine neuroblastoma cells, $1 \times 106$ cells in DMEM media with $50 \%$ matrigel in 100 ul without fetal bovine serum and without antibiotics were subcutaneously injected on the right flanks. After 12 days, tumor growth can be seen and tumors were measured with a caliper. When tumors reached $100 \mathrm{~mm}^{3}$ in size, mice were divided into two groups with 8-10 mice in each group.

\section{Intratumoral delivery of siRNA}

SiRNA-HDAC8 (S88696), Sense Sequence: (5'----3') CGACGGAAAUUUGACCGUAtt

Antisense Sequence:

UACGGUCAAAUUUCCGUCGca

SiRNA-MYC (S70224), Sense Sequence: (5'------3') AGGUAGUGAUCCUCAAAAAtt 
Negative control \#2 siRNA (\#4390846), and Lipofectamine RNAi Max (\#13778075) were purchased from Life Technologies/Ambion

A total of $3 \mathrm{nmol}$ Negative control siRNA or $3 \mathrm{nmol}$ combinations of siRNA-MYC + siRNA-HDAC8 were mixed with Lipofectamine RNAi max (Liposome) in DMEM media without fetal bovine serum and without antibiotic. siRNA complexed with Lipofectamine in a volume of $30 \mathrm{ul}$ was delivered into tumors by intratumoral injection every third day. Tumors were measured every second day with a caliper and mice were weighed every third day. Tumor volume was calculator with a formula, $\mathrm{V}=$ Length $X$ width $^{2} / 2$. Experiment was stopped when the control tumors treated with negative control siRNA reached a tumor burden volume of $1200 \mathrm{~mm}^{3}$. Mice were euthanized by $\mathrm{CO}_{2}$ inhalation 2 days after last treatment with siRNA. Tumors were removed and weighed. Tumors were frozen in liquid nitrogen and stored at $-80^{\circ} \mathrm{C}$ freezers until used for preparation of tumor extracts for ELISA.

\section{Tumor extract preparation}

Tumors treated with NC-siRNA or with combined siRNA-HDAC8 + siRNA-MYC were cut into small pieces and homogenized in assay buffer in a glass homogenizer. Assay buffer as described by Khandelia et al. [15], consisted of $20 \mathrm{mM}$ Tris- $\mathrm{HCL} \mathrm{pH} 7.5,150 \mathrm{mM} \mathrm{NaCl}, 5 \mathrm{mM}$ EDTA, $10 \%$ glycerol, $1 \%$ Nonidet P40, and protease inhibitor cocktail from Sigma (P8340). Protein concentrations were determined using Pierce's BCA Assay as per the manufacturer's instructions.

\section{HDAC8 and c-MYC protein quantitation via ELISA}

HDAC8 and c-MYC proteins were quantitated in extracts prepared from tumors via ELISA. 100ug protein per sample was mixed with $0.02 \mathrm{M}$ carbonate coating buffer ( $\mathrm{pH}$ 9.5) and added to 96-well BD-Falcon ELISA plates for protein binding. Samples were incubated at $4^{\circ} \mathrm{C}$ for $15 \mathrm{~h}$. Wells were blocked with $10 \%$ FBS in PBS and treated with primary antibodies (diluted 1:30) specific for HDAC8 (SC11405) or c-MYC (SC-798), and incubated at $37^{\circ} \mathrm{C}$ for $2 \mathrm{~h}$. Samples were washed with PBS $+0.05 \%$ Tween, treated with goat anti-rabbit IgG-HRP secondary antibody (diluted 1:500), and incubated at $37^{\circ} \mathrm{C}$ for $1 \mathrm{~h}$. Wells were washed and treated with substrate TMB and incubated at room temperature for $30 \mathrm{~min}$, and then the reaction was stopped with $2 \mathrm{~N} \mathrm{H}_{2} \mathrm{SO}_{4}$. Samples were read at $450 \mathrm{~nm}$ in a plate reader.

\section{Statistical analysis}

Error bars represent standard error of the mean (SEM) from 5-8 biological replicates from 2 independent experiments. $P$-values were calculated using $T$-Test (2 tailed, 3 samples, unequal variance) and $p<0.05$ was considered statistically significant.

\section{Results}

\section{Neuroblastoma Tumor treatment with the combination of siRNA-HDAC 8 + siRNA-MYC}

We explored the therapeutic effect of treatment with the combination of SiRNA-HDAC8 + siRNA-MYC on neuroblastoma tumor xenografts in mice.

Mouse neuroblastoma cells were inoculated subcutaneously into 16, 6-week-old female A/J mice. Tumors were formed, with an average volume of 100 $\mathrm{mm}^{3} 12$ days after cells were inoculated. These mice were divided into two groups of 8 mice. Then, one group of mice treated with a $3 \mathrm{nmol}$ negative control siRNA (NCsiRNA) and other group of mice were treated with a 3 nmol combination of siRNA-HDAC8 + siRNA-MYC. siRNAs was inoculated into tumors by intratumoral injections every third day.

Tumor growth was measured every two days. The rate of tumor growth was significantly decreased following treatment with combined siRNA-MYC + siRNA-HDAC8 compared to treatment with control negative siRNA (Figure 1A).

When the control tumors reached an average volume of more than $1200 \mathrm{~mm}^{3}$, the mice were euthanized and the tumors were removed (Figure 1B, C) The average weight of 8 tumors treated with the combination of siRNA-HDAC8 + siRNA-MYC was decreased 5-fold $(0.186$ g) compared to the average weight of control tumors (1 g) treated with NC-siRNA (Figure 1D). The tumor xenograft experiment was repeated with 8 mice treated with NCsiRNA and 8 mice treated with a combination of siRNAHDAC8+SIRNA-MYC. 


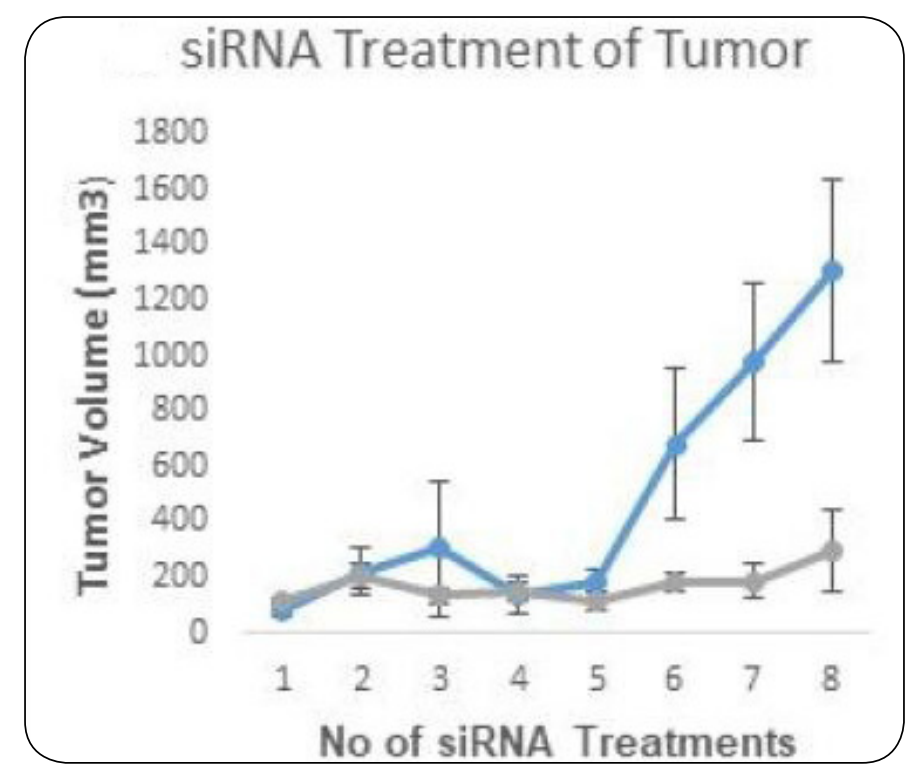

Figure 1A: Effect of combination of siRNA on the growth of neuroblastoma Tumor. Murine neuroblastoma cells, $1 \times 106$ cells in DMEM media with $50 \%$ matrigel in 100 ul were subcutaneously injected on the right flanks. After 12 days, tumor growth can be seen and tumors were measured with a caliper. When tumors reached $100 \mathrm{~mm}^{3}$ in size, mice were divided into two groups with 8 mice in each group. Negative control siRNA or combination of siRNA-MYC + siRNA-HDAC8 was mixed with Lipofectamine RNAi max (Liposome) in DMEM media without fetal bovine serum and without antibiotic. siRNA complexed with Lipofectamine in a volume of 30 ul was delivered into tumors by intratumoral injection every third day. Tumors were measured every second day with a caliper and mice were weighed every third day. Tumor volume was calculator with a formula, $V=$ Length $\times$ width $^{2} / 2$. The numbers on X-axis show the number of siRNA treatments. Control tumor growth is shown by diamond $(\wedge)$ markers and the growth of tumors treated with siRNAs is shown by round $(0)$ markers. SEM bars represent the standard deviation from 8 mice at each point $P^{*}<0.031$.

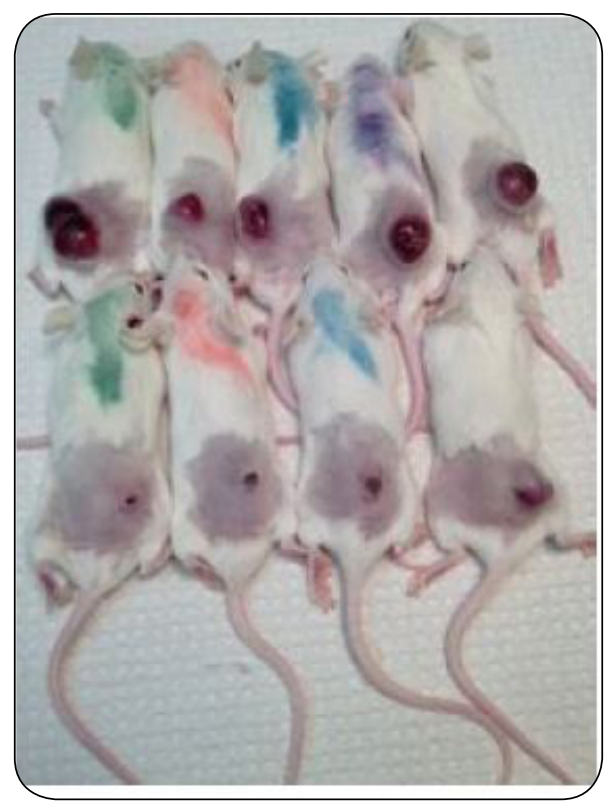

Figure 1B: Mice with control and combination of siRNA treated Tumors. Top row of mice with control tumors treated with NC siRNA and the bottom row of mice with tumors treated with the combination of siRNA-MYC + SiRNA-HDAC. 


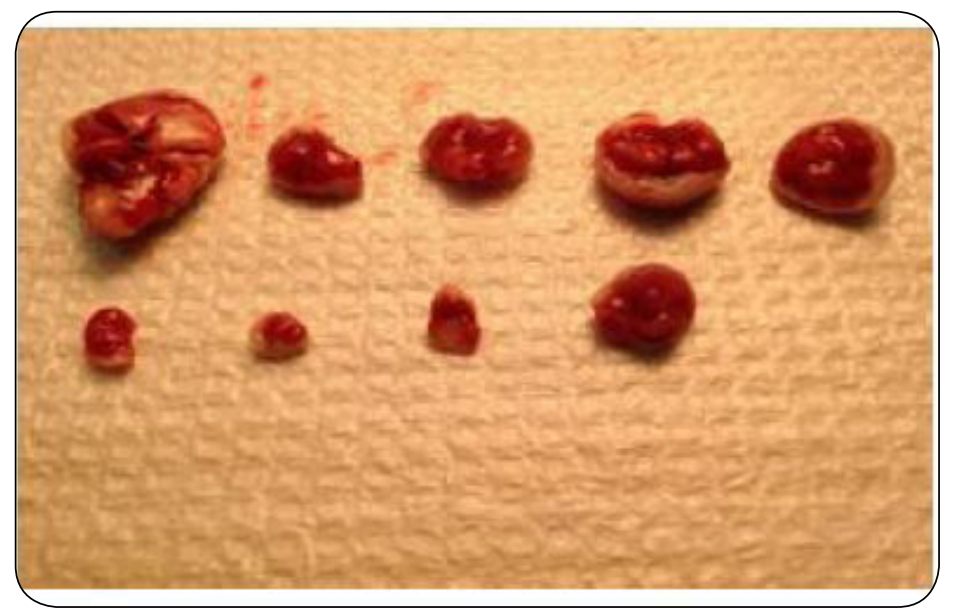

Figure 1C: Tumors from control and combination of siRNA treated mice. Top row representative control tumors treated with NC-siRNA and the bottom row representative tumors treated with the combination of siRNA-MYC + siRNA-HDAC8.

\section{The Quantitation of the targets Hdac8 and Myc in Tumors}

To confirm the effectiveness of the siRNA treatment, Hdac8 and Myc were quantified by ELISA in extracts prepared from the tumors. The levels of the targets Hdac8

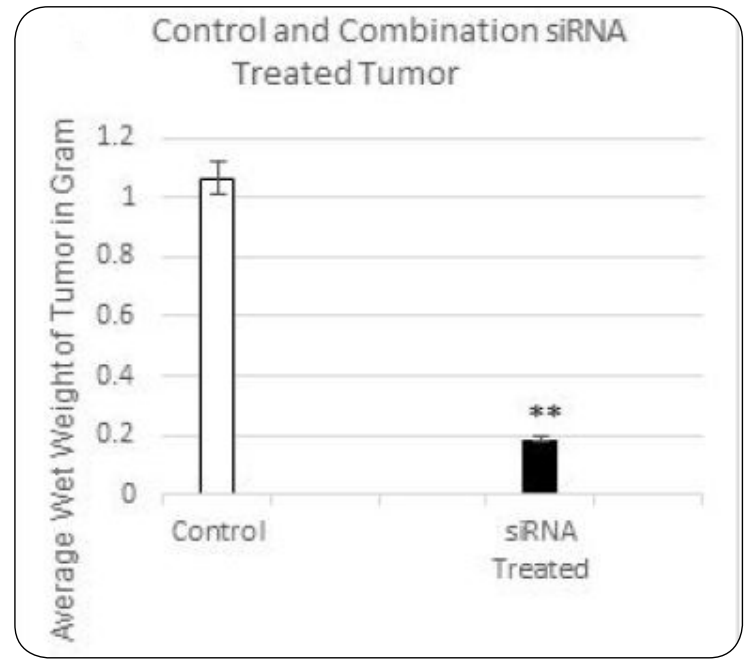

Figure 1D: Weights of control and combination of siRNA treated Tumors. Average of 8 control tumors was 1 gram and average of 8 tumors treated with combination of siRNAMYC + siRNA-HDAC8 was 0.186 gram. SEM bars represent the standard deviation from 8 tumors $P^{* *}<0.004$.

and Myc were decreased by $85 \%$ and $64 \%$, respectively, in tumors treated with the combination of siRNA-HDAC8 +siRNA-MYC compared to tumors treated with NC-siRNA (Figure $2 A$ and $B$ ). These results indicate that a decrease in the levels of the tumoral targets HDAC8 and Myc caused the inhibition of tumor growth.

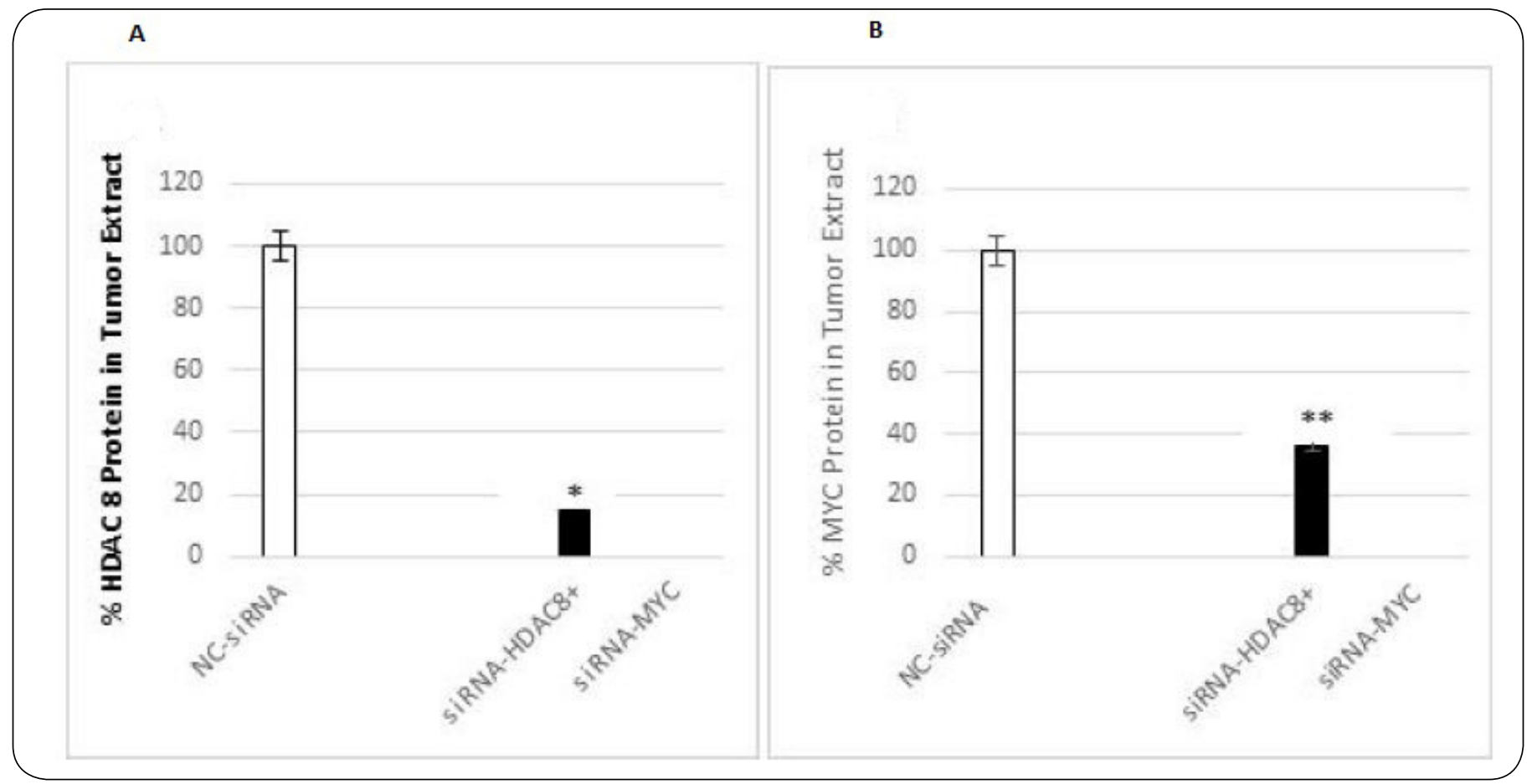

Figure 2A and B: Quantitation of Myc and Hdac8 proteins from control and combination of siRNA treated tumors. Tumor targets Hdac8 and Myc proteins were quantitated by ELISA in extracts prepared from 3 tumors treated with negative control siRNA and 3 tumors treated with the combination of siRNA-HDAC $8+$ siRNA-MYC. Average targets Hdac8 [Figure 2A] and Myc [Figure 2B] proteins were decreased by $85 \%$ and $65 \%$ in tumors treated with the combination of siRNA-HDAC8 + siRNA-MYC compared to tumors treated with NC-siRNA. SEM bars represent the standard deviation from 3 tumors $\left(P^{*}<0.030, P^{* *}<0.01\right)$. 


\section{Discussion}

In targeted cancer therapy miRNA and siRNA are used as therapeutic agents to knock down the expression of target oncogenic genes in order to inhibit the growth of cancer cells.

As a therapeutic approach, siRNA has been used to target the genes encoding overexpressed cancer proteins in order to inhibit cancer cell growth in vitro and to inhibit tumor growth in vivo in mouse models of the following cancers: breast cancer, glioma and colon cancer [10-13].

MYC and HDAC8 each have been shown to contribute to neuroblastoma tumorigenesis.

Our previous findings in neuroblastoma cells indicated targeting both Myc and Hdac8 with a combination of siRNAs inhibited cell growth by $86 \%$ and was more effective than either siRNA alone [14].

Here we confirm these findings in a mouse neuroblastoma xenograft model, where tumor growth was reduced by $80 \%$ following intratumoral delivery of a combination of siRNAs targeting both Myc and Hdac8 at the same time.

These results indicate that the targeting of Myc and Hdac8 specifically caused in the inhibition of tumor growth in vivo. Therefore, targeting simultaneously more than one gene product which is responsible for the tumorigenesis will be an effective therapy for xenograft tumor model. Our data is consistent with that of Tiejun et al. [16], who determined that multi-target siRNAs showed better silencing effects on three targets compared with single target siRNA. These data indicate that knockdown of more than one target, which are involved in tumorigenesis; same time will be more effective than the knockdown of single target.

MYC and HDAC8 are overexpressed in several cancers, such as in lung, breast and colon cancers; therefore, the combination of siRNA therapy will be tested in the xenograft tumor models of these cancers to inhibit the growth of the tumors.

In this study siRNAs were delivered into tumors by intratumoral injections. This decreased the levels of Myc and Hdac8 and inhibited the growth of tumor. However, this delivery route is not feasible in the clinical therapy; therefore a systemic IV delivery will be tested and improved.
Our results show proof of the concept for using of the combination of SiRNA-MYC + siRNA-HDAC8 for neuroblastoma therapy.

\section{Conclusion}

Neuroblastoma is the most frequently diagnosed extracranial solid tumor in children. These tumors account for $15 \%$ of childhood deaths from cancer. Survival in oneyear-old children is $<30 \%$ despite aggressive therapies. We are using mouse neuroblastoma tumor model to understand the role of Myc and Hdac8 in tumorigenesis.

We previously found that in vitro, single-agent siRNAHDAC8 or siRNA-MYC inhibited cell proliferation by 40$50 \%$; however, treatment with the combination of siRNAMYC + siRNA-HDAC8 inhibited cell proliferation by $86 \%$ [14]. We further confirmed these findings in an animal model, and verified that tumor growth can be inhibited in a neuroblastoma xenograft mouse model when tumors are treated with a combination of SiRNA-MYC and SiRNAHDAC8.

\section{Declarations}

Ethics approval: Mice experiments were performed with the approval of the institutional Animal Care and Use Committee, IACUC at Nanospectra Biosciences Inc. Houston, Texas.

Competing interest: We declare no conflict of interest.

Funding: TMC Internist, Houston, Texas provided partial funding for the chemical supplies used in this project.

Authors' contribution: Author planned, designed and performed the experiments and wrote the manuscript.

Acknowledgements: The author would like to thank Dr. George Calin and associates at MD Anderson Cancer Center, Houston, Texas, for access to the luminometer plate reader.

Conflict of interest: There is no conflict of interest.

\section{References}

1. World Health Organization. 2014. Chapter 5.16.

2. MarisJM, Hogarty MD, Bagatell R, etal. Neuroblastoma. 
Lancet. 2007;369(9579):2106-2120. Doi: https:// dx.doi.org/10.1016/S0140-6736(07)60983-0

3. Chang TC, Yu D, Lee YS, et al. Widespread microRNA repression by Myc contributes to tumorigenesis. Nat Genet. 2008;40(1):43-50. Doi: https://dx.doi. org/10.1038/ng.2007.30

4. O'Donnell KA, Wentzel EA, Zeller KI, et al. C-mycregulated micrornas modulate e2f1 expression. Nature. 2005;435(7043):839-843. Doi: https://dx.doi. org/10.1038/nature03677

5. Dang CV. C-myc target genes involved in cell growth, apoptosis, and metabolism. Mol Cell Biol. 1999;19(1):111. Doi: https://dx.doi.org/10.1128/mcb.19.1.1

6. Richon VM, Sandhoff TW, Rifkind RA, et al. Histone deacetylase inhibitor selectively induces p21WAF1 expression and gene-associated histone acetylation. Proc Natl Acad Sci USA. 2000;97(18):10014-10019. Doi: https://dx.doi.org/10.1073/pnas.180316197

7. Oehme I, Deubzer HE, Wegener D, et al. Histone deacetylase 8 in neuroblastoma tumorigenesis. Clin Cancer Res. 2009;15(1):91-99. Doi: https://dx.doi. org/10.1158/1078-0432.CCR-08-0684

8. Glick RD, Swendeman SL, Coffey DC, et al. Hybrid polar histone deacetylase inhibitor induces apoptosis and cd95/cd95 ligand expression in human neuroblastoma. Cancer Res. 1999;59(17):4392-4399.

9. Coffey $D C$, Kutko MC, Glick RD, et al. The histone deacetylase inhibitor, CBHA, inhibits growth of human neuroblastoma xenografts in vivo, alone and synergistically with all-trans retinoic acid. Cancer Res. 2001;61(9):3591-3594.

10. Liang $Y$, Gao H, Lin SY, et al. Sirna-based targeting of cyclin e overexpression inhibits breast cancer cell growth and suppresses tumor development in breast cancermouse model.PLOSONE.2010;5(9):e12860.Doi: https://dx.doi.org/10.1371/journal.pone.0012860
11. Uchida H, Tanaka T, Sasaki K, et al. Adenovirusmediated transfer of sirna against survivin induced apoptosis and attenuated tumor cell growth in vitro and in vivo. Molecular Therapy. 2004;10(1):162-171. Doi: https://dx.doi.org/10.1016/j.ymthe.2004.05.006

12. Oh BY, Lee RA, Kim KH. Sirna targeting livin decreases tumor in a xenograft model for colon cancer. World Journal of Gastroenterology. 2011;17(20):2563-2571. Doi: https://dx.doi.org/10.3748/wjg.v17.i20.2563

13. Zhang $\mathrm{X}, \mathrm{Ge} \mathrm{YL}$, Tian $\mathrm{RH}$. The knockdown of c-myc expression by RNAi inhibits cell proliferation in human colon cancer HT-29 cells in vitro and in vivo. Cell Mol Biol Lett. 2009;14(2):305-318. Doi: https:// dx.doi.org/10.2478/s11658-009-0001-9

14. Prashad N. Mir-665 targets c-myc and hdac8 to inhibit murine neuroblastoma cell growth. Oncotarget. 2018;9(69):33186-33201. Doi: https://dx.doi. org/10.18632/oncotarget.26046

15. Khandelia P, Yap K, Makeyev EV. Streamlined platform for short hairpin RNA interference and transgenesis in cultured mammalian cells. Proc Natl Acad Sci USA. 2011;108(31):12799-12804. Doi: https://dx.doi. org/10.1073/pnas.1103532108

16. Li T, Xue Y, Wang G, et al. Multi-target sirna: therapeutic strategy for hepatocellular carcinoma. J Cancer. 2016;7(10):1317-1327. Doi: https://dx.doi. org/10.7150/jca.15157 reproduction in any medium, provided the original work is properly cited. 